\title{
A Brief Introduction of Symbol Application in Animation Design
}

\author{
Hongjuan Sun \\ College of Art and Design \\ Huanghe Science and Technology College \\ Zhengzhou, China 450063
}

\begin{abstract}
Animation is a comprehensive artistic class, an artistic expression, combining drawing, cartoon, film, digital media, photography, music and literature as a whole. As a media for visual communication, animation itself is a symbol, a symbol expressed through images, colors, light and shadow and auditory sense.
\end{abstract}

Keywords—visual symbol; animation images; animation scenes; audio-visual

\section{INTRODUCTION}

The symbol is a special medium for human beings to know the outward objects, and it is also an expression approach of humans' thoughts and emotions. Due to its proper directive, it becomes an important path for audiences' knowledge transmission and interaction; humans' consciousness realm is a very world of symbols. Animation is a special visual art which mixes art and sciences together, by an exaggerated and suppositional world to mirror the actual phenomenon, and a special medium to express humans' inner world. Apart from the visual symbols, animation also has unique auditory symbols, which enables it to be the medium of information transmission and the carrier of social culture from visual and auditory level.

\section{SYMBOLIC MEANING OF ANIMATION IMAGES}

From the day that animation came into being, the images of animation also changes. Those animation images that we are very familiar with not only brought about joy and delight, but also make us closely knitted to animation. The molding style changed from the mass and public taste to the highlighting of personal style. Nowadays, the animation is not restricted to one single expression form anymore.

By abundant animation approaches, especially exaggeration and abstraction to code at random, the animation images can explicitly convey the compiler's intention to the audiences.

\section{A. Animation Images: Existence of Symbols}

What is symbol? A symbol is identification with a certain meaning, usually refers to literature, language, code, mathematical symbol, chemical symbol and traffic sign etc. Cassirer, a semiotics aesthetician deemed that "Art can be defined as a symbolic language," it is a formal symbol to express our thoughts and emotions. Animation is as a special artistic expression form, so the roles in animation are also a kind of lansign; by performing these roles, the idea of the animation can be conveyed to the audiences.

A galloping buffalo was drawn in Altamira Cave of Spain; its well-shaped and vivid image is very impressive. The most impressive part is that its tail and legs were repeatedly painted, and it brings a sense of motion to the viewers. Such instances also exist in China. The representative of Majiayao Culture of The Neolithic Age, "Colored Dancing Pottery basin" is the same as the buffalo of Altamira Cave. There are three groups of dancing figures, five dancers for each on the basin; they are dancing hand by hand. The arms of the outermost figures of every group are also painted twice to reflect the swing of the dancers. These examples are the most primitive animation images. With the development of motion picture technique, animation is becoming increasingly popular among audiences. The first thing that attracts the audiences' attention of the animation is the roles. The images are symbols; the animation itself is a special artistic form of symbols. The art style of an animation determines the features of the image and the meaning that is to be conveyed to the audiences.

After determining the types of role, the designer of the animation is to design the role model; vitality of the role has direct impact on the enjoyment of the entire animation. When designing the animation roles, usually special visual forms should be adopted since it is easy to be identified and remembered; it is also easy to stimulate the audiences' senses. The roles should have special modeling elements and be pithy and general. The designer should shape the roles that meet the requirements of the animation from the whole plot and all characters' features of the animation; it is very important to select types of roles in animation creation. There are many types of animation roles; however, there must be a role that can catch audiences' attention in a successful animation, such as an (a) antagonist, hero, lovely role etc. Different models bring different psychological experience to the audiences, and this is the function the symbol of animation images.

\section{B. Typical Animation Image: Text of Symbols}

The lansign in our daily life is usually in the form of text, while the animation adopts the performance of animation role to convey information. Animation image is the symbolic text of animation art. For example, as long as we mention Disney 
Animation, Mickey Mouse may first enter our mind; therefore, Mickey Mouse, the cartoon image, is a symbolic text.

The stories of traditional Chinese animation usually are adapted from Chinese myths, and the expression forms are varied, including ink painting, paper cutting and puppet; therefore, these animation images have typical Chinese features. This is also an expression of animation image symbols. Making havoc in Heaven is adapted from Journey to the West. The protagonist of the animation is Sun Wukong, whose image design takes in the element of Chinese Opera Facial Designs, which is a typical image in China and is very impressive in the audiences' mind.

\section{SyMBOLIC FORMATION OF ANIMATION COLORS}

As one of the artistic modeling elements, color is also a symbol in semiology because color is endowed with symbolism, meaning and emotion; therefore, colors have different symbolic meanings in different artistic context. Colors have emotional meanings, and different colors hues, tonality, and brightness can create different emotional feelings; for example, red symbolizes hue, joy and optimism; green life, youth and environmental protection; black death, concealment and nobility; white purity, divineness and tidiness. Different colors can make people think of different things and scenes.

Color symbol is the necessary element to compose visual cognition system of animation. The design of roles and scenes cannot be separated from color, and color is a significant composing part of artistic design of animation, which enables the animation have strong visual shock. The signified of color in animation means to have an impression on color for the audiences; the signifier of color in animation refers to impressing the audiences psychologically and making them have imagination about color. For example, in Three Monks, clothes of little three monks are jacinth because jacinth is the symbol of loveliness and youth, which complies with the ages and characters of the little monks. Audiences can feel the features of the roles through different colors. In the creation of animation, except from modeling different roles, color symbols can also render and contrast the atmosphere the entire animation. In the Disney animation, The Lion King, there are dense selva, colorful flowers and clear waterfall when modeling Simba's growing environment, while Scar's surroundings is always black and gloomy caves even the rules the whole animal world. The prosperous prairie turns into a sea of fire, and the tonality is always blue and choky. The colors are identical to the plot and atmosphere of the story; by exaggerated application of colors, the atmosphere of the animation is rendered.

Apart from that, colors also have contrasting function in expressing peoples' inner emotion and expectation. In the animation work of Hayao Miyazaki, Spirited Away, when Chihiro enters the tunnel with her parents, the black and long tunnel reflects Chihiro's fear. After a long pass of the tunnel, there is a little bit of light at the end of the tunnel, which indicates that Chihiro is eager to walk out of the tunnel and see what the other sides of the tunnel looks like.

Animations of different themes and nationalities have distinct style difference in color application. The theme of
Disney animation is mainly about fairy tales, so most animations are rich-colored, sprightly and lovely; traditional Chinese animation is influenced by Chinese traditional arts; therefore, the color contrast is distinct and it has unique features of Chinese traditional painting; the themes of Hayao Miyazaki's animations are mainly environmental protection, vitality, dreams and life, and the color of the animations is mainly cyan, bright and pure; there are much clear water and prosperous woods in his animations.

The color symbol of animations is a significant element to compose animation; the creator demonstrates his/her creation intention by color matching and tonality contrast. Apart from the modeling, colors can also convey the creator's thoughts to the audiences.

\section{DEMONSTRATION OF LIGHT AND SHADOW SYMBOLS}

Light can be divided into natural light and artificial light; natural light refers to the light produced by the sun or through reflection or refraction, while artificial light means the illuminant produced by human beings in indoor environment.

The objects will have shadow when illuminated and different angles of illuminant may produce different shadows.

We can see this colorful world and beautiful environment around us because there is light. If there is no light, all will be in darkness and nothing will be seen. The light is of help for the expression of material texture; apart from that light can also bring us the sense of chill and warmth, false and true, strong and weak. The shadow itself has metaphor and symbolic meaning and renders a mysterious atmosphere visually. Light and shadow play a vital role in animation films.

Apart from modeling spaciousness, third dimension, sense of structure and layering, light and shadow as a symbol can also render an atmosphere for the scene and the audiences can have a psychological emotion when watching it; the light and shadow can be used to create a horrible and mysterious effect. Light and shadow is also very important to characterize animation roles; in The Lion King, we can see clear light and shadow distinct when characterizing the protagonist and the negative characters. When Simba appears, the sun is always shining rightly; while Scar is always living in a dark shadow. The animation demonstrates the two characters' features by light and shadow in a subtle way.

No light no shadow, light and shadow are like twins. As a symbol, light and shadow not models spaciousness in the scene, but also implies the audiences' mental feelings.

\section{FUNCTIONS OF AUdITORY SYMBOLS IN ANIMATION}

Animation conveys information by moving pictures and sound. Visual and auditory language is a unique technology of films and it is also a symbol coding system in mass media. Animation is a category of film. In visual and auditory languages, "listening" is an important element. Apart from the attractive scenes, an animation is also an auditory enjoyment. As a symbolic expression, visual and auditory language has its own unique functions. 
Lange deems that up to now, the most advanced and shocking symbolic design created by human beings is language. For human's auditory sense, things that are easy to be remembered by our brains are language and sound, and this is what an animation role conveys to the audiences. From the perspective of semiology, the signifier of auditory symbols is human voice, sound, music, and its signified means that the sound brings the audiences psychological and physical feelings, which make the animation more vivid and bring the audiences to the plots easier.

Auditory symbols have rhythm and impressiceness; the sound can generalize the theme, contrast the atmosphere and highlight the style of the animation. The sound can be expressed by the auditory images, such as the sound of the roles, including dialogues and monologues, sound effect in the environment and background music.

Apart from interpreting the scenes, sound can also be a drama element. The film often characterizes figures and characters through sound; besides, sound can convey contents to the audiences beyond the scenes to strengthen the rhythm of interaction between sound and scenes. The different combinations among forms and contents of sound and scenes could create various effects full of with expressive force and deepen the contents and connotations of scenes. The big success of Three Monks is not only owing to its unique artistic style, but also successful music and acoustics. There is no one dialogue or monologue, but traditional Chinese percussion instruments such as drum, cymbals enriches the color of music. Music takes a vital role in the film, such as characterizing the figures, rendering emotions and environment and promoting the development of plots. Different characters are characterized by different instruments and different instruments reflect the different characters of figures. The ringing voice of banhu fiddle can reflect the smartness and loveliness of the little monk; the changeable sound of zhuihu fuddle is employed to depict the tall monk's acerbity and the vigorous sound of pipe is used to describe the fat monk's simplicity and honesty; therefore, the artistic effect of "less is more" is achieved, which increases the enjoyment and abundance of the animation.

Everyone has their own speech habits and language style, as well as the animation characters. For example, the famous sentence "Truth is always only one" in Detective Conan can always incurs people to think of the character Conan who is shrunken by the large glasses. Lansign can be remembered by the audiences through numerous repetitions; however, simple and boring repetitions do not work, and it is must be applied in certain circumstances. Apart from the language of the characters, the theme song can also have such a function. The famous song Love Me Tonight of The Lion King is such as song; as long as comes into our ears, the love story between Simba and Nala.

Sound, that controls the rhythm of film, renders the atmosphere, expresses the inner world of characters and demonstrates national cultural connotations, is the crucial factor in the animation films. In modern animation works, sound can contrast the scenes of the animation, and it has been independent from the scene as an independent symbol with its own aesthetic value and research significance.

\section{CONCLUSION}

All in all, the symbol design of animation has to meet three requirements: the first one is all the visual elements presented in the animation, i.e., the animation figures' images; the second one is the animation itself and the third one is transmission channel, i.e., the media, such as the theaters and televisions. The audiences can understand the deepened meaning through these requirements. The animation symbols can not only make the audiences feel the images, colors, light and shadow and auditory sense, it can also present symbolic and abstract information.

\section{REFERENCES}

[1] Jia Fou \& Lu Shengzhang, Understanding Animation [M] Communication University of China Press, 2008.

[2] Yu Hong, Scene Design of Television Animation-Blending Historicality and Contemporaneity, Mastering the Integral Shape of Art [J]. Science and Technology Innovation Herald, 2009.

[3] Han Xiao, Layout Design in Animated Film and Video [M]. China Ocean Press, 2005, 07.

[4] Li Bin, Insights into Sign: An Ontological Analysis of Media Content [M]. Fudan University Press, 2003.

[5] Gu Chen, Study on Visual Linguistic Symbol in Animation [J]. Pioneering with Science \& Technology Monthly, 2010.03. 